\title{
Regulation of thermogenesis in Brown and Beige Adipose Tissues
}

\author{
Xinwei Li ${ }^{1 *}$, Yue Chen ${ }^{2}$, Hongxia Liư ${ }^{1}$, Yuanyuan Tong ${ }^{1}$ and Chaoyong He*1 \\ ${ }^{1}$ Department of Pharmacology and State Key Laboratary of Natural Medicines, China Pharmaceutical University, China \\ ${ }^{2}$ Department of General Surgery, Hefei Second People's Hospital, China
}

*Corresponding author: Chaoyong He and Xinwei Li, Department of Pharmacology and State Key Laboratary of Natural Medicines, China Pharmaceutical University, Nanjing, China.

To Cite This Article: Xinwei Li, Yue Chen, Hongxia Liu, Yuanyuan Tong, Chaoyong He. Regulation of thermogenesis in Brown and Beige Adipose Tissues. 2020 - 8(6). AJBSR.MS.ID.001328.

DOI: 10.34297/AJBSR.2020.08.001328.

Received: 畊April 17, 2020; Published: 眥 May 14, 2020

\begin{abstract}
Obesity and related metabolic syndromes are among the top public health issues worldwide. The current strategy is mainly based on controlling food intake, unable to achieve the optimal treatment effect. How to augment energy expenditure becomes a new focus. White adipose tissue (WAT) is a classic energy storage depot, whereas brown adipose tissue (BAT) dissipates energy through thermogenesis. Browning of WAT facilitates the transformation from WAT into beige adipose tissue acquiring modest thermogenic activity. Enhancement of thermogenic function of both BAT and beige adipose tissue highlights a novel perspective to dissipate excess stored energy and reduce lipid deposition. In this review, we systematically summarize the current regulatory mechanisms of thermogenesis revealed in BAT and beige adipose tissue, including the thermogenic function of mitochondria, the crosstalk between macrophages and adipocytes, endoplasmic reticulum stress as well as proteasome activity. Additionally, we discuss the underlying signaling network to regulate relevant gene expression involved in these four aspects so as to provide a better understanding for therapeutic strategy design to treat obesity and related metabolic diseases.
\end{abstract}

Keywords: WAT, BAT, Beige Adipose Tissue, Thermogenesis

\section{Introduction}

Obesity and related metabolic syndromes have been one of the most concerned public health problems worldwide [1]. With improved life quality and the change of lifestyle such as sedentariness, staying up late and lacking of exercise, the incidence of obesity and its complications is increasing dramatically in recent years. Currently, the treatment strategy is mainly based on controlling food intake. However, obesity is caused by the longterm energy intake exceeding energy consumption. Hence, how to augment energy expenditure becomes a new focus for obesity treatment.

The main feature of obesity at cellular level is increased fat accumulation and adipocytes hypertrophy. According to the structure and function of adipocytes, adipose tissue is classified into white adipose tissue (WAT) and brown adipose tissue (BAT). WAT with unilocular lipid droplets and few mitochondria is the main depot of excess energy in the form of 95\% Triglyceride and a small amount of cholesterol. Different from WAT, BAT shows more intense mitochondria, innervation and vascular content, which mainly produces heat in a uncoupled protein 1 (UCP1) dependent manner to maintain body temperature following cold stimulation
[2]. This indicates that the improvement of BAT thermogenic activity could be the key target for energy expenditure to treat obesity [3]. Whereas a declined weight ratio and excessive lipid deposition of BAT in obese patients, the effect to treat obesity by enhancing the thermogenic function of BAT is largely limited [4].

In recent decades, "browning" or "beigeing" of WAT draws attention as an alternative strategy to increase energy consumption and to prevent weight gain [5]. This procedure facilitates the transformation from WAT into adipose tissue carrying partial molecular features of BAT. The transformed adipose tissue is named as beige or brite adipose tissue, which possesses abundant cristaedense mitochondria showing increased UCP1 expression level and multilocular lipid droplets [6]. The transformation is strongly induced in response to specific environmental conditions and external factors, including chronic cold acclimation, exercise, cancer cachexia, tissue injury and long-term treatment with peroxisome proliferator-activated receptor $\gamma(\operatorname{PPAR} \gamma)$ or $\beta 3$-adrenergic receptor ( $\beta 3$-AR) agonists [7]. This metabolic transformation indicates therapeutic potential to prevent dietary related obesity and increase the sensitivity to insulin treatment [8]. 


\section{Mechanisms Regulating Thermogenesis in Brown and Beige Adipose Tissues}

Transformation from WAT into beige adipose tissue to treat obesity is challenging. It's well known that classical brown adipocytes still retain multilocular lipid morphology days after the withdrawal of thermogenic stimulation [12]. In contrast, beige adipocytes rapidly diminish the transient partial features of BAT and restores the unilocular lipid morphology [12]. The discrepancy highlights the need for a better understanding of the mechanisms regulating the thermogenesis of BAT and beige adipose tissue. Here we explore and summarize the mechanisms mainly related to the thermogenic function of mitochondria, the infiltrated macrophages, ER (endoplasmic reticulum) stress and proteasomal activity in brown and beige adipocytes.

\section{Thermogenic Function of Mitochondria}

Mitochondria play important roles in thermogenic process in both brown and beige adipocytes. Nevertheless, these two adipocyte types show divergence in thermogenic function and amounts of mitochondria. Mitochondrial biogenesis and clearance are tightly controlled to maintain the optimal mitochondria amounts in adipose tissues. Mitochondria biogenesis is remarkably activated in both BAT and beige adipose tissue with external stimuli e.g. chronic cold exposure. But mitochondria clearance plays a dominant role in beige adipocytes following the withdrawal of stimulation [9] (Figure 1).

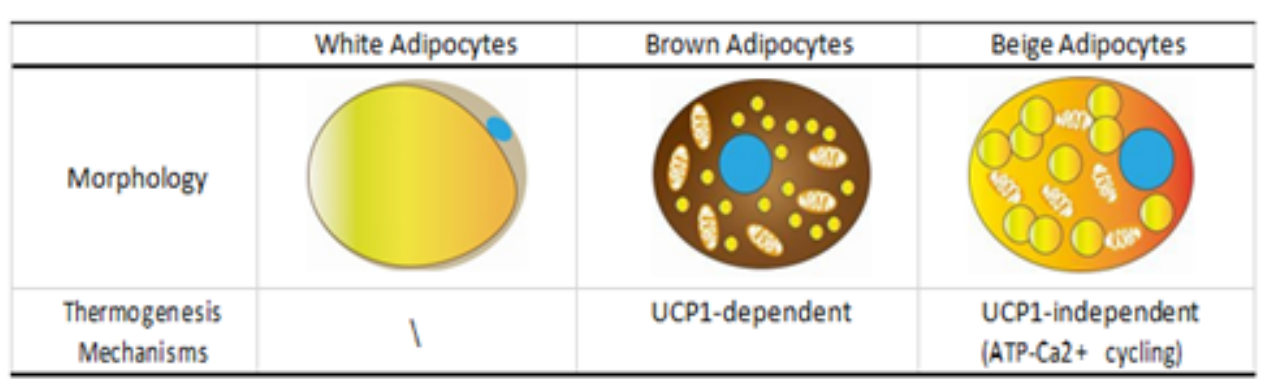

Figure 1: The differences of white, brown and beige adipocytes.

Thermogenic function of mitochondria is closely associated with UCP1, which is highly expressed in classical BAT to uncouple the mitochondrial proton gradient from adenosine triphosphate (ATP) synthesis in order to dissipate energy and produce heat [10]. The UCP1 expression level is finely controlled by transcriptional regulation. Once stimulated by cold exposure, sympathetic neurons in BAT release norepinephrine (NE), which activates $\beta 3$-AR to further, trigger intracellular cyclic AMP (cAMP) signaling. The latter activates cAMP dependent protein kinase A (PKA) to phosphorylate downstream proteins such as cAMP responsive element binding protein (CREB), a major agonist to trigger the transcription of UCP1 gene [11,12]. PRD1-BF1-RIZ1 homologous domain-containing protein 16 (PRDM16) is a critical regulator controlling brown fat determination by stimulating brown fat-selective gene expression, while suppressing the expression of genes selective for white fat [13]. PRDM16 binds to CCAAT/enhancer binding protein- $\beta$ (C/ EBP- $\beta$ ) to promote the expression of transcriptional factor PPAR $\gamma$ and PPAR $\gamma$ coactivator- $1 \alpha$ (PGC- $1 \alpha)$ that then stimulate the transcription of specific-thermogenic genes including UCP1 [14]. However, the expression of UCP1 in beige adipose tissue is lower than BAT [15], and chronic treatment with $\beta 3$-AR agonist increases respiration of epididymal WAT in UCP1-/- mice [16]. These indicate browning of WAT is not completely UCP1 dependent. It could be due to Sarco/endoplasmic reticulum Ca2+-ATPase 2b (SERCA2b)/ Ryanodine receptor 2 (RyR2)-dependent Ca2+ cycling, which retains high cold tolerance in the UCP1-/- beige adipose tissue [17]. Increased intracellular calcium circulation following
NE stimulation activates ATP synthesis via a voltage-dependent anion channel and mitochondrial calcium uniporter [18]. Through the SERCA2b/RyR2 signaling pathway, beige adipose tissue dynamically dissipates glucose to maintain glycolysis, tricarboxylic acid metabolism and pyruvate dehydrogenase activity for ATPdependent metabolic thermogenesis to generate heat and increase energy expenditure [19].

Brown adipocytes show robust activation of mitochondrial biogenesis with thermogenic stimulation. Following NE combination with $\beta 3-\mathrm{AR}$, activating transcription factor 2 (ATF2) is up-regulated by p38/mitogen-activated protein kinase (p38/MAPK), which subsequently triggers the transcriptional activity of PGC- $1 \alpha / \beta 20$. The latter induces the expression of nuclear respiratory factor $1 / 2(N R F 1 / 2)$ [21-23] and activation of mitochondrial transcriptional factor A and B (mtTFA and mtTFB) to enhance mitochondrial replication [23]. Similarly, WAT activates mitochondrial biogenesis at a more moderate level once exposed to external thermogenic stimulation, facilitating the transformation to beige adipose tissue [24].

Brown adipocytes retain their multilocular lipid morphology and highly activated mitochondria biogenesis following withdrawal of cold stimulation. However, beige adipocytes gradually recover unilocular lipid droplets and activate mitophagy to eliminate excess or damaged mitochondria [25]. The reduced mitochondrial membrane potential leads to stabilization of phosphatase and tensin homolog-induced putative kinase 1 (PINK) and subsequent 
recruitment of ubiquitin ligase parkin from cytoplasm to the outer membrane of the damaged mitochondria. In addition, the withdrawal of cold stimulation triggers parkin recruitment to the outer membrane of excess mitochondria [25]. Parkin mediates ubiquitination of target proteins of outer membrane following phosphorylation by PINK and promotes the release of autophagic "eat me" signal [26]. Next, selective autophagy adaptor proteins including nuclear dot protein (NDP52), optineurin (OTEN) and SQSTM1/p62 form autophagy receptors and link the ubiquitinated protein to microtubule-associated protein 1 light chain 3 (LC3) on the outer membrane to turn on mitophagy to clear the excess and damaged mitochondria [27].

\section{The Crosstalk between Adipocytes and Macrophages}

The crosstalk between adipocytes and macrophages nearby plays an important role in obesity development and thermogenic activation [25,28]. In obese status, white adipocytes release inflammatory chemokines such as interferon $\gamma$ (IFN- $\gamma$ )and monocyte chemotactic protein 1 (MCP1) to promote monocyte infiltration and polarization of proinflammatory macrophages [29]. Adipose tissue macrophage (ATM) show a M1-like phenotype, of which the biological markers are however not completely identical as those participating in anti-infectious immune responses [30].
ATM produces pro-inflammatory cytokines, metalloproteinases (MMP) and reactive oxygen species (ROS), which together contribute to adipocyte hypertrophy, adipose tissue dysfunction and insulin resistance [31]. The pro-inflammatory cytokines such as tumor necrosis factor $\alpha(\mathrm{TNF} \alpha)$ and interleukin-1 $\beta$ (IL-1 $\beta$ ) inhibit UCP1 expression in both BAT and WAT [32]. Mechanistically, the pro-inflammation factors activate extracellular signal-related kinase (ERK) signaling to suppress the activity of UCP1 promoter and mRNA expression [33].

Conversely, BAT-derived pro-inflammatory IL-6 increases plasmic and BAT fibroblast growth gactor-21 (FGF21) levels, which facilitate WAT browning and UCP1 expression. IL-6 also directly induces M2 polarization validated by the detection of expression markers of M2 macrophages in WAT from cold-stimulated mice [34]. While primarily exhibiting anti-inflammatory phenotype [35], M2 macrophages release NE to activate $\beta 3-A R$ pathway [36] and secrete PPAR $\gamma$ ligands [37] to promote the browning of white adipocytes.

Hence, the infiltrated macrophage phenotype and the crosstalk with adipocytes affect the balance between adipogenesis and thermogenesis of both BAT and WAT (Figure 2).

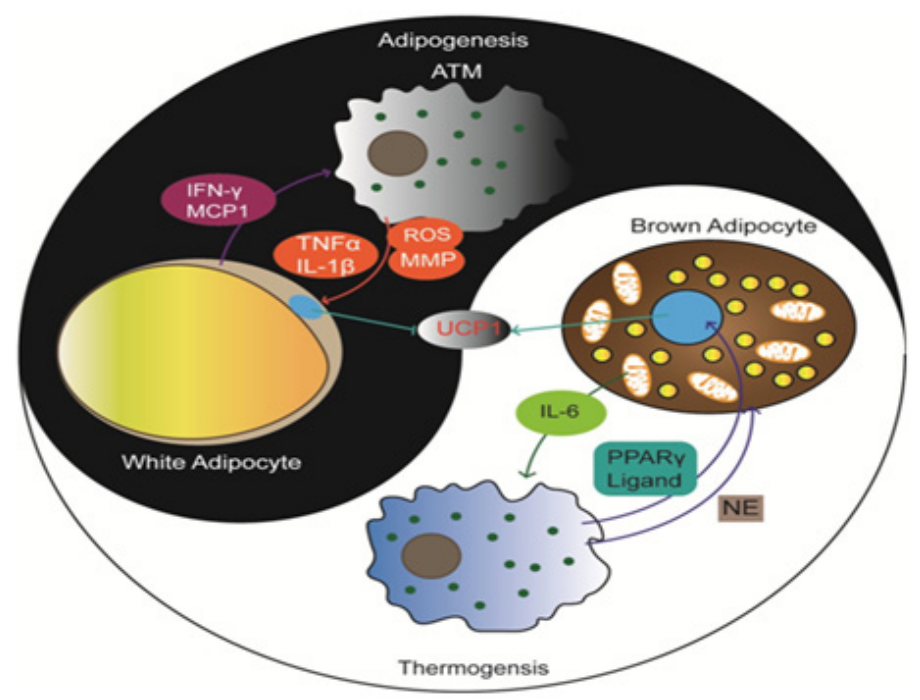

Figure 2: The balance of adipogenesis and thermogenesis influenced by the crosstalk between adipocytes and macrophages. ATM releases proinflammatory cytokines, MMP and ROS to induce lipid accumulation in WAT. M2 macrophages release NE and PPARy to induce thermogenesis in BAT. Conversely, IFN-y and MCP1 from WAT and IL-6 from BAT induce corresponding macrophages polarization.

\section{ER stress}

ER is the largest organelle in eukaryotic cells, important for lipid synthesis [38]. Excessive accumulation of lipid, oxygen deficiency and ischemia reperfusion cause ER stress and aggregation of misfolded or unfolded proteins, thereby inducing unfolded protein response (UPR) [38]. UPR signal induced proteins include transmembrane protein kinase 1 (IRE-1), activating transcription factor 6 (ATF-6) and protein kinase R like endoplasmic reticulum kinase (PERK) [39]. IRE1 activates the c-Jun N-terminal kinase
(JNK) signaling pathway and upregulates the expression of X-box binding protein 1 (XBP1), as well as downregulates the expression of anti-apoptotic gene BCL-2 [40]. The activated ATF6 transfers into the nucleus to promote the transcription of ER stress response element (ERSE), which activates downstream XBP1 and CCAAT enhancer binding protein homologous protein (CHOP) and leads to apoptosis [41]. PERK activates PERK-eukaryotic initiation factor 2a (eIF2a)-phosphorylation activating transcription factor-4 (ATF4) axis to trigger the expression of CHOP [42], which further induces cell death [43]. 
ER stress plays important roles during obesity progression [7]. In ob/ob mice, hypertrophic adipocytes and excessive accumulation of fat are related with ER stress and increased CHOP expression [44]. Phosphorylation of IRE1 $\alpha$ and JNK as an ERstress-response indicator, is markedly augmented in subcutaneous and retroperitoneal adipose tissues from rats fed with margarine [45]. In line with this, obese patients show significantly activated ER stress in WAT, including the activation of the ATF6 pathway and phosphorylation of eIF2 $\alpha$ [46]. Those protein involvement cause insulin resistance by reduced insulin receptor substrate (IRS1) in response to insulin in adipose tissue. In addition, FFAs (free fatty acids) are released during fat mobilization into the circulation, which enter non-adipose tissues, such as the skeletal muscle and liver. This causes the ectopic accumulation of lipid and affects physiological functions of these organs, including the promotion of insulin desensitisation in other organs [47].

ER stress is also an important regulator for the thermogenic process of BAT. Obesity-induced ER stress reduces BAT thermogenesis by inhibition of mtDNA expression and enhancement of CHOP-mediated apoptosis of brown adipocytes [48]. Conversely, the IRE1 $\alpha$ loss promotes energy expenditure through promoting UCP1 expression to enhance the thermogenic activity of brown as well as beige adipose tissue [49]. And Sirt1 (sirtuin type 1) binds to ATF4 and directly reduces the expression in BAT, leading to CHOP increasing and apoptosis [50]. All these suggest targeting ER stress and downstream could a potential avenue to improve thermogenic activity of adipose tissues (Figure 3).

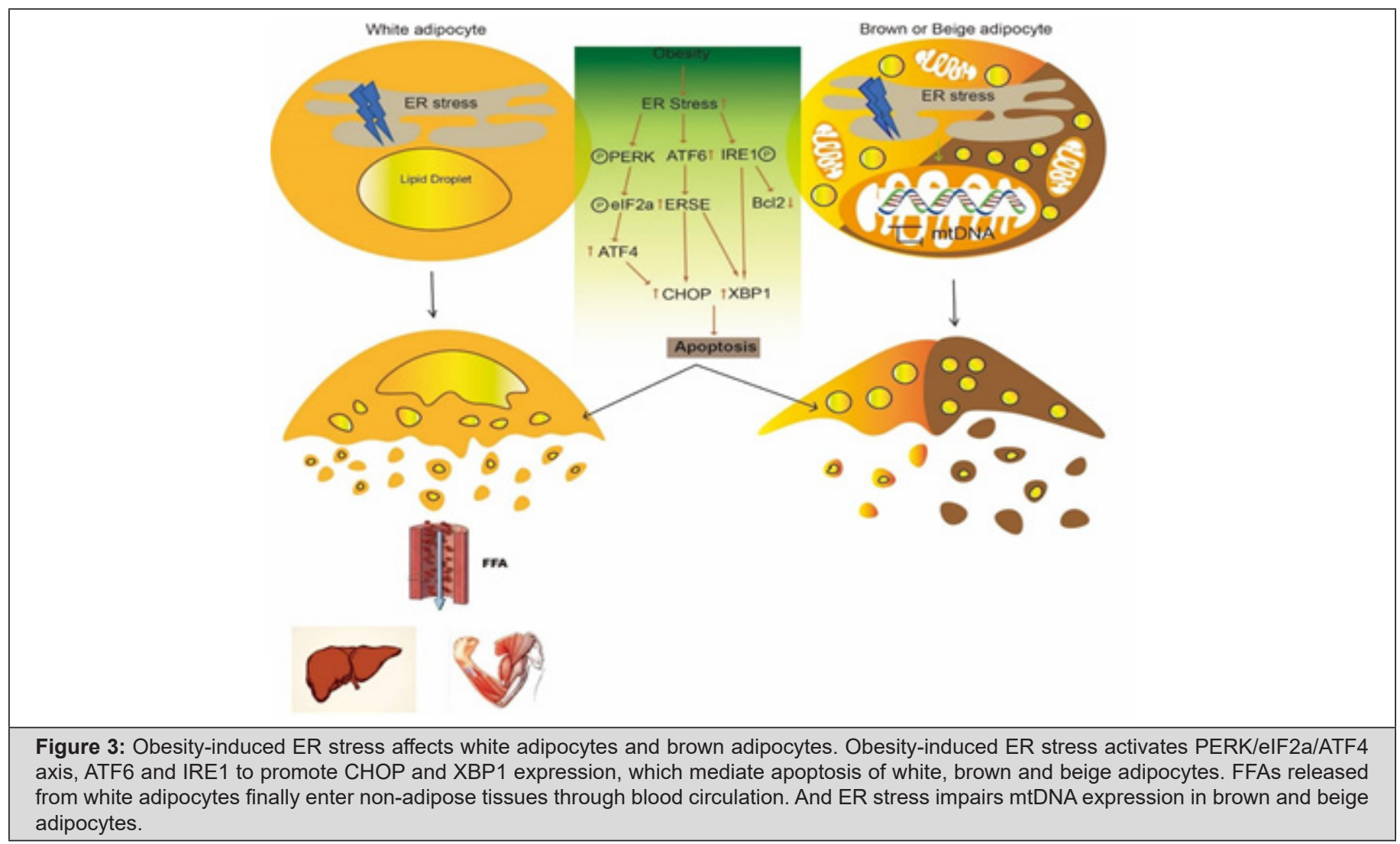

\section{Proteasomal activity}

As the site of protein and lipid synthesis, the ER is a critical organelle for the maintenance of metabolic and homeostatic balance [39]. Obesity related ER stress is combated by the UPR, as well as by ER-associated protein degradation (ERAD) of damaged or dispensable proteins via the ubiquitin-proteasome system [51]. The proteasomal activity is higher in BAT under the $4{ }^{\circ} \mathrm{C}$ or room temperature $\left(22^{\circ} \mathrm{C}\right)$ condition than thermoneutrality $\left(30^{\circ} \mathrm{C}\right)$, which is independent of changes in the mRNA expression of UCP1 or PPARGC1A. Therefore, proteasome function in BAT metabolic adaptation, especially cold-induced thermogenesis [52]. Recently, an ER-localized transcription factor nuclear factor erythroid 2-like 1 (Nfe2l1, also known as Nrf1) has been confirmed as a critical driver of this process. Nrf1-KO mice in cold condition show lower mRNA expression of genes encoding proteasomal subunits and proteasomal activity, compared to WT mice [52]. As reported, proteasomal inactivity is associated with K48-linked accumulation and proteasomal degradation of protein ubiquitination forms Lys48(K48)-modification ubiquitin chains [53]. Compared to WT controls, the higher abundance of ubiquitinated proteins in BAT of Nrf1-KO mice including K48, K11 and K708 ubiquitin linkages, and many mitochondrial respiration proteins including UCP1 are also hyper-ubiquitinated [52]. Therefore, Nrf1 as an ER-localized TF to increase proteasomal activity maintaining the ER homeostasis and high thermogenic activity by regulated ubiquitination numbers of related thermogenic proteins (Figure 4). 


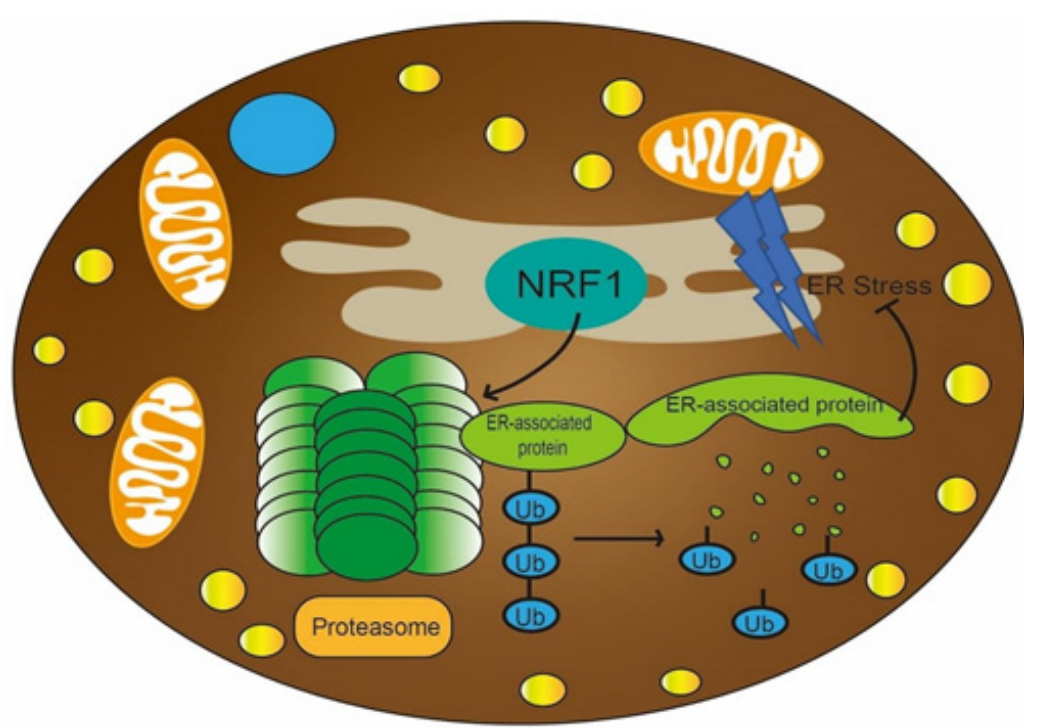

Figure 4: Brown adipocyte health are critical to secure proteostasis and metabolic integrity during states of high activity. ER-localized transcription factor nuclear factor Nrf1 in BAT increase proteasomal activity, and then reduce ER stress to maintain the ER homeostasis and cellular integrity, as well as reduce hyper-ubiquitinated proteins to enable the proper turnover of ubiquitinated thermogenic proteins, such as UCP1.

Signaling network regulating thermogenic process of BAT and beige adipose tissue

Thermogenic function of mitochondria, the infiltrated macrophage, ER stress and proteasomal activity are important aspects for thermogenic process of BAT and beige adipose tissue.
Hence, a systematic review about the signaling network regulating the expression levels of molecules participating in these aspects is highly required. Here we explore and summarize the current knowledge of cellular signaling pathways involved in thermogenic process of adipocytes (Table 1).

\begin{tabular}{|c|c|c|c|c|c|}
\hline Signaling Pathway & $\begin{array}{c}\text { Type of } \\
\text { Transcrption Factor }\end{array}$ & $\begin{array}{l}\text { Genetyping of } \\
\text { Mice }\end{array}$ & $\begin{array}{c}\text { Main results on BAT } \\
\text { Thermogenesis and WAT } \\
\text { Browning }\end{array}$ & Mechanism & Function \\
\hline Notch Signaling & Hes & Notch-knockout & $\begin{array}{c}\text { UCP1+ multicellular cells in WAT } \\
\uparrow \text { Thermogenesis marker UCP1 } \\
\text { Cidea } \uparrow \text { Beige cell marker CD137 } \\
\uparrow \text { Mitochondrial gene COX7a } \uparrow\end{array}$ & $\begin{array}{l}\text { Thermogenic function } \\
\text { of mitochondria }\end{array}$ & $\begin{array}{l}\text { Hes directly bind to the } \\
\text { promoter of PRDM16 } \\
\text { and PGC- } 1 \alpha \text { to repress } \\
\text { thermogenesis. }\end{array}$ \\
\hline \multirow{2}{*}{ JAK/STAT Signaling } & STAT1/STAT2 & IFN $\alpha$-activation & $\begin{array}{c}\text { Thermogenesis marker UCP1 } \downarrow \\
\text { Mitochondrial genes Cox7a } \downarrow \\
\text { Oxygen consumption } \downarrow\end{array}$ & $\begin{array}{l}\text { Thermogenic function } \\
\text { of mitochondria }\end{array}$ & $\begin{array}{l}\text { IFN-activated } \\
\text { STAT1/2 may directly } \\
\text { repress the UCP1 } \\
\text { transcription. }\end{array}$ \\
\hline & STAT3 & Tyk2-knockout & $\begin{array}{c}\text { Thermogenesis marker } \\
\text { UCP1 Cidea PRDM16 BAT } \\
\text { development related RNA C/ } \\
\text { EBP } \beta \text { and PPAR } \gamma \downarrow\end{array}$ & $\begin{array}{l}\text { Thermogenic function } \\
\text { of mitochondria }\end{array}$ & $\begin{array}{l}\text { STAT3 forms a } \\
\text { complex with PRDM16 } \\
\text { and enhance the } \\
\text { stability of PRDM16 }\end{array}$ \\
\hline \multirow{3}{*}{ TLR-IRF Signaling } & IRF3 & IRF3-knockout & $\begin{array}{c}\text { WAT Mass } \downarrow \text { (HFD) } \\
\text { Inflammatory genes } \downarrow \text { (HFD) } \\
\text { Energy expenditure } \uparrow \\
\text { UCP1+ multicellular cells } \uparrow \\
\text { Thermogenesis marker } \uparrow\end{array}$ & $\begin{array}{l}\text { Thermogenic function } \\
\text { of mitochondria The } \\
\text { crosstalk between } \\
\text { adipocytes and } \\
\text { macrophages }\end{array}$ & $\begin{array}{l}\text { (1) Alter macrophage } \\
\text { polarization state; } \\
\text { (2) IRF3 activation } \\
\text { may be a mediator of } \\
\text { TLR4 signaling. }\end{array}$ \\
\hline & & $\begin{array}{c}\text { IRF4- } \\
\text { overexpression }\end{array}$ & $\begin{array}{l}\text { Lipid droplets } \downarrow \text { Thermogenesis } \\
\text { marker UCP1 PRDM16 PGC-1 } \downarrow\end{array}$ & \multirow[b]{2}{*}{$\begin{array}{l}\text { Thermogenic function } \\
\text { of mitochondria }\end{array}$} & \multirow[b]{2}{*}{$\begin{array}{l}\text { IRF4 directly binds to } \\
\text { the promoter of UCP1. }\end{array}$} \\
\hline & IRF4 & IRF4-knockout & $\begin{array}{c}\text { Maintain body temperature } \\
\text { Lipid content in BAT } \uparrow \\
\text { Thermogenesis marker } \downarrow \\
\text { Mitochondrial respiration } \downarrow\end{array}$ & & \\
\hline
\end{tabular}




\section{Notch Signaling}

Notch signaling is activated by the interaction between Notch ligands and receptors, leading to notch intracellular section (NCID) released into the cytoplasm, which further translocates into the cell nucleus. Next, the formation of NCID/CSL transcription activation complex activates Hes and Hey, two transcription inhibitory factors from alkaline-screw-ring-screw family [54]. Notch signaling affects thermogenic function of adipocytes through regulation of mitochondrial function. Evidently, the adiposespecific Notch1- knockout (KO) mice show higher expression levels of BAT related molecules (including UCP1, CIDEA, PRDM16, PPARGC1A) and beige cells markers, like (CD137, also called TNFRSF9 or TBX1), furthermore mitochondrial genes COX7A, compared to WT (wild-type) mice. The Notch-KO mice have higher rectal temperatures than their WT siblings. Conversely, activation of NICD or over expression of target genes suppress the expression of the thermogenesis associated UCP1, PRDM16 and PPARGC1A but increase the expression of $H E S$ and $H E Y$. In addition, the metabolic rate and body temperature in activation NICD of mice are also lower than those in WT mice. Mechanistically, it results from the activation of the canonical Notch downstream Hes, which directly binds to the promoters of PRDM16 and PPARGC1A to repress their transcription, thereby blocking brown or beige related expression profile55.

\section{JAK/STAT Signaling}

JAK (Janus kinase) phosphorylates STAT (Signal transducer and activator of transcription) family members, leading to STATs dimerization. Then STATs dimers translocate to the nucleus to trigger the expression of targeted genes. Among the STAT family members STAT1, STAT2 and STAT3 have reported to regulate the process of adipocytes differentiation and thermogenesis,

\section{STAT1/2}

Activated type I IFN signaling upon viral infection rapidly activates STAT1 and STAT2, two key effectors of the IFN response56. Recently, IFN $\alpha$ is demonstrated to inhibit preadipocytes 3T3-L1 differentiation during the early stage of adipogenesis by arresting the cell cycle at the G0/G1 phase and suppress the expression of PPAR $\gamma$ and C/EBP $\alpha$ through JAK mediated STAT1 phosphorylation [57]. Interestingly, IFNa treated brown adipocyte precursor cells show dramatic decrease of thermogenic markers UCP1 and CIDEA, mitochondrial gene COX7A together with mitochondrial encoded genes $m t$-CYTB and $m t$-CO157 [58]. These alterations result in disorganized cristae and reduced oxygen consumption but not mitochondrial biogenesis. The effects are reversed by ectopic activation of PRDM16, which decreases both the mRNA and protein levels of STAT1 and STAT2. It is speculated that IFNactivated STAT1/2 directly represses the transcription of UCP 1 and mitochondrial-encoded genes [58].

\section{STAT3}

As opposed to STAT1/2, STAT3 is critical for the differentiation of brown adipocytes, which is phosphorylated by Tyk2, a JAK family member. Tyk2 is decreased in obese mice and patients [59]. Tyk2-KO mice show lower energy expenditure and lower body temperature in cold condition. The decreased mRNA levels of UCP1, CIDEA and PRDM16 and the disorganized cristae of mitochondria are observed, as well as reduce mitochondrial oxygen consumption [59]. Exogenous STAT3 expression is used to restore the differentiation of Tyk2-/- preadipocytes, which formed complex with PRDM16 to enhance the stability of PRDM16, a master regulator of BAT differentiation. IN addition, exotic STAT3 in Tyk2-/- mice show normal levels of insulin and significantly lower body weights. Furthermore, BAT thermogenesis related RNA (UCP1 and Cidea) and BAT development related RNA (C/EBP $\beta$ and PPAR $\gamma$ ) are also increased. However, rosiglitazone (a PPAR $\gamma$ agonist stabilizes PRDM16 protein)-induced the PRDM16 up-regulation at protein level is not observed in STAT3-/- brown preadipocytes [59]. Hence, STAT3 play a critical role in regulation of BAT differentiation and combat obesity.

\section{TLR-IRF Signaling}

The Toll-like receptor (TLR) family induces type 1 IFN response via activation of FN-regulatory factor (IRF) to trigger immune responses against microbial pathogens. Accumulating evidence demonstrates that this signaling axis affects thermogenesis of brown and beige adipocytes by reprogramming the interaction with invaded immune cells as well as mitochondria function.

\section{IRF3}

IRF3 is constitutively expressed in a variety of cells, mainly in cytoplasm. TLR4 can induce phosphorylation of IRF3, which forms dimer and shifts to the nucleus, and combines with other transcription factors to induce expression of IFN $\alpha / \beta$ and IFN stimulating genes [60]. LPS activated TLR4 signaling activated reduces thermogenesis related with reduced body temperature and brown-specific genes such as UCP1 and PRDM16, as well as promote obesity related with ER stress and increased CHOP expression [61]. Conversely, IRF3-KO mice show enhanced oxygen consumption, heat output and energy expenditure. Expression level of UCP1 is upregulated during the development of beige adipocytes and increase thermogenic marker genes CIDEA and PRDM16 in inguinal WAT of IRF3-KO mice on high-fat diet, but not in classic interscapular BAT [62]. In addition, after 1 month at thermoneutrality, IRF3-KO mice increase oxygen consumption as well as UCP1 and PRDM16 expression in the iWAT but not in BAT compared to WT mice. This suggests IRF3 plays an inhibitory role in temperature-independent browning of WAT [62].

High-fat diet results in excessive lipid accumulation in adipocytes and intra-adipose inflammation [32]. Wang, X. A et 
al. found that IRF3-/- mice show reduced fat mass as well as inflammatory gene expression in adipose tissues especially in inguinal WAT and liver but increased lean mass. Furthermore, IRF3 participates in the alteration of the polarization state of the infiltrated macrophages, which is demonstrated by reduced M1like macrophages and increased M2-like macrophages in inguinal WAT of IRF3-KO mice [62].

\section{IRF4}

IRF4 also participates in the negative regulation of TLR signaling as well as the regulation of immune cell cells differentiation and macrophages polarization [63]. Recently, IRF4 is reported to regulate energy metabolism. Kong et al. demonstrated that both IRF4 mRNA and protein levels increase in BAT, inguinal WAT, and epididymal WAT of C57BL/6 mice treated with cold stimulation or administration of the 33 -agonist [64]. Targeted expression of IRF4 causes enhanced energy expenditure and leanness. Increased IRF4 also promotes thermogenesis in BAT and induces the transcription of thermogenic genes UCP1, PRDM16 and PPARGC1A. Although over expression of IRF4 mice can maintain body temperature in cold condition though increasing uncoupled respiration, though a mild change of mitochondrial encoding protein expression [64]. Conversely, IRF4-KO mice show increased lipid content in BAT and reduced energy expenditure and predisposes to obesity. IRF4 loss results in reduction of mitochondrial respiration related with reduced gene expression such as COXI, and detrimental mitochondrial structure as well as decrease of UCP1, PRDM16 and PPARGC1A. Mechanistically, IRF4 induces the expression of PGC- $1 \alpha$ and PRDM16 and interacts with PGC- $1 \alpha$, driving UCP1 expression. Cold stimulation, $\beta$-agonist, or forced expression of PGC- 1 a do not cause thermogenic gene expression in the absence of IRF464. Together, these results validate IRF4 as a transcriptional driver of thermogenic gene expression to maintain mitochondria function.

\section{Conclusion}

The thermogenesis in BAT and beige adipose tissue is finely controlled by the thermogenic function of mitochondria, the crosstalk between macrophages and adipocytes, ER stress as well as proteasome activity. Notch, JAK/STAT and TLR-IRF signaling together with Nrf1 compose a complex signaling network to dynamically regulate critical genes expression playing in the above four aspects. Enhancement of thermogenesis in BAT and beige adipose tissue is a potential strategy avenue to obtain optimal balance between energy storage and consumption for obesity treatment. However, application of pharmacological intervention to promote thermogenic function of BAT and beige adipose tissue and beigeing of WAT remains dim. As such, further studies to explore novel compounds facilitating the thermogenic process of adipose tissues would aid in the design of more efficient treatment strategies.

\section{Declaration of Interests}

The authors declare no competing interests.

\section{Acknowledgment}

This research was supported by the National Natural Science Foundation of China (81670425), Jiangsu Specially-Appointed Professors Program, the Jiangsu Innovative and Entrepreneurial Program, the "Double First Class" University Project of China Pharmaceutical University (CPU2018GF04), and the Open Project of the State Key Laboratory of Natural Medicines of China Pharmaceutical University (SKLNMZZCX201809) through funding awarded to Chaoyong He.

\section{References}

1. Wang H, Qiang L, Farmer SR (2008) Identification of a domain within peroxisome proliferator-activated receptor gamma regulating expression of a group of genes containing fibroblast growth factor 21 that are selectively repressed by SIRT1 in adipocytes. Mol Cell Biol 28(1): 188-200.

2. Rosen ED, Spiegelman BM (2014) What we talk about when we talk about fat. Cell 156(1-2): 20-44.

3. Farmer SR (2009) Obesity: Be cool, lose weight. Nature 458(7240): 839840 .

4. Chechi K, Nedergaard J, Richard D (2014) Brown adipose tissue as an anti-obesity tissue in humans. Obes Rev 15(2): 92-106.

5. Kozak LP (2010) Brown fat and the myth of diet-induced thermogenesis. Cell Metab 11(4): 263-267.

6. Seale P, Conroe HM, Estall J, Kajimura S, Frontini A, et al. (2011) Prdm16 determines the thermogenic program of subcutaneous white adipose tissue in mice. J Clin Invest 121(1): 96-105.

7. Fu S, Watkins SM, Hotamisligil GS (2012) The role of endoplasmic reticulum in hepatic lipid homeostasis and stress signaling. Cell Metab 15(5): 623-634.

8. Fan L, Ye H, Wan Y, Qin L, Zhu L, et al. (2019) Adaptor protein APPL1 coordinates HDAC3 to modulate brown adipose tissue thermogenesis in mice. Metabolism 100: 153955 .

9. Altshuler-Keylin S, Shinoda K, Hasegawa Y, et al. (2016) Beige Adipocyte Maintenance Is Regulated by Autophagy-Induced Mitochondrial Clearance. Cell Metab 24(3): 402-419.

10. Golozoubova V, Hohtola E, Matthias A, Jacobsson A, Cannon B, Nedergaard J (2001) Only UCP1 can mediate adaptive nonshivering thermogenesis in the cold. FASEB J 15(11): 2048-2050.

11. Cannon B, Nedergaard J (2004) Brown adipose tissue: function and physiological significance. Physiol Rev 84(1): 277-359.

12. Bauters D, Cobbaut M, Geys L, Van Lint J, Hemmeryckx B, Lijnen HR (2017) Loss of ADAMTS5 enhances brown adipose tissue mass and promotes browning of white adipose tissue via CREB signaling. Mol Metab 6(7): 715-724.

13. Kajimura S, Seale P, Tomaru T, Cooper MP, Ruas JL, et al. (2008) Regulation of the brown and white fat gene programs through a PRDM16/CtBP transcriptional complex. Genes Dev 22(10): 1397-1409.

14. Kajimura S, Seale P, Kubota K, Lunsford E, Frangioni JV, et al. (2009) Initiation of myoblast to brown fat switch by a PRDM16-C/EBP-beta transcriptional complex. Nature 460(7259): 1154-1158. 
15. Nedergaard J, Cannon B (2013) UCP1 mRNA does not produce heat Biochim Biophys Acta 1831(5): 943-949.

16. Granneman JG, Burnazi M, Zhu Z, Schwamb LA (2003) White adipose tissue contributes to UCP1-independent thermogenesis. Am J Physiol Endocrinol Metab 285(6): E1230-E1236.

17. Ikeda K, Kang Q, Yoneshiro T, Camporez JP, Maki H, et al. (2017) UCP1independent signaling involving SERCA2b-mediated calcium cycling regulates beige fat thermogenesis and systemic glucose homeostasis. Nat Med 23(12): 1454-1465.

18. Denton RM (2009) Regulation of mitochondrial dehydrogenases by calcium ions. Biochim Biophys Acta 1787(11): 1309-1316.

19. Kramarova TV, Shabalina IG, Andersson U, Westerberg R, Carlberg I, et al. (2008) Mitochondrial ATP synthase levels in brown adipose tissue are governed by the c-Fo subunit P1 isoform. FASEB J 22(1): 55-63.

20. Cao W, Daniel KW, Robidoux J, Puigserver P, Medvedev AV, et al. (2004) p38 mitogen-activated protein kinase is the central regulator of cyclic AMP-dependent transcription of the brown fat uncoupling protein 1 gene. Mol Cell Biol 24(7): 3057-3067.

21. MacGarvey NC, Suliman HB, Bartz RR, , Fu P, Withers CM, et al. (2012) Activation of mitochondrial biogenesis by heme oxygenase-1mediated NF-E2-related factor-2 induction rescues mice from lethal Staphylococcus aureus sepsis. Am J Respir Crit Care Med 185(8): 851861.

22. Piantadosi CA, Suliman HB (2006) Mitochondrial transcription factor A induction by redox activation of nuclear respiratory factor 1 . J Biol Chem 281(1): 324-333.

23. Gleyzer N, Vercauteren K, Scarpulla RC (2005) Control of mitochondrial transcription specificity factors (TFB1M and TFB2M) by nuclear respiratory factors (NRF-1 and NRF-2) and PGC-1 family coactivators. Mol Cell Biol 25(4): 1354-1366.

24. Fisher FM, Kleiner S, Douris N, Fox EC, Mepani RJ, et al. (2012) FGF21 regulates $\mathrm{PGC}-1$ alpha and browning of white adipose tissues in adaptive thermogenesis. Genes Dev 26(3): 271-281.

25. Ikeda K, Maretich P, Kajimura S (2018) The Common and Distinct Features of Brown and Beige Adipocytes. Trends Endocrinol Metab 29(3): 191-200.

26. Geisler S, Holmstrom KM, Skujat D, Fiesel FC, Rothfuss OC, et al. (2010) PINK1/Parkin-mediated mitophagy is dependent on VDAC1 and p62/ SQSTM1. Nat Cell Biol 12(2): 119-131.

27. Richter B, Sliter DA, Herhaus L, Stolz A, Wang C, et al. (2016) Phosphorylation of OPTN by TBK1 enhances its binding to Ub chains and promotes selective autophagy of damaged mitochondria. Proc Natl Acad Sci USA 113(15): 4039-4044.

28. Villarroya F, Cereijo R, Villarroya J, Giralt M (2017) Brown adipose tissue as a secretory organ. Nat Rev Endocrinol 13(1): 26-35

29. Lackey DE, Olefsky JM (2016) Regulation of metabolism by the innate immune system. Nat Rev Endocrinol 12(1): 15-28.

30. Kratz M, Coats BR, Hisert KB, Hagman D, Mutskov V, et al. (2014) Metabolic dysfunction drives a mechanistically distinct pro inflammatory phenotype in adipose tissue macrophages. Cell Metab 20(4): 614-625.

31. Guzik TJ, Skiba DS, Touyz RM, Harrison DG (2017) The role of infiltrating immune cells in dysfunctional adipose tissue. Cardiovasc Res 113(9): 1009-1023.

32. Sakamoto T, Nitta T, Maruno K, Yeh YS, Kuwata H, et al. (2016) Macrophage infiltration into obese adipose tissues suppresses the induction of UCP1 level in mice. Am J Physiol Endocrinol Metab 310(8): E676-E687.

33. Sakamoto T, Takahashi N, Sawaragi Y, Naknukool S, Yu R, et al. (2013) Inflammation induced by RAW macrophages suppresses UCP1 mRNA induction via ERK activation in 10T1/2 adipocytes. Am J Physiol Cell Physiol 304(8): C729-C738.
34. Stanford KI, Middelbeek RJ, Townsend KL, Nygaard EB, Hitchcox KM, et al. (2013) Brown adipose tissue regulates glucose homeostasis and insulin sensitivity. J Clin Invest 123(1): 215-223.

35. Qiu Y, Nguyen KD, Odegaard JI, Cui X, Tian X, et al. (2014) Eosinophils and type 2 cytokine signaling in macrophages orchestrate development of functional beige fat. Cell 157(6): 1292-1308.

36. Nguyen KD, Qiu YF, Cui XJ, Goh YP, Mwangi J, et al. (2011) Alternatively activated macrophages produce catecholamines to sustain adaptive thermogenesis. Nature 480(7375): U104-U272.

37. Lee YH, Kim SN, Kwon HJ, Maddipati KR, Granneman JG (2016) Adipogenic role of alternatively activated macrophages in betaadrenergic remodeling of white adipose tissue. Am J Physiol Regul Integr Comp Physiol 310(1): R55-R65.

38. Kelly E, Greene CM, Carroll TP, McElvaney NG, O’Neill SJ (2009) Selenoprotein S/SEPS1 modifies endoplasmic reticulum stress in Z variant alpha1-antitrypsin deficiency. J Biol Chem 284(25): 1689116897.

39. Amen OM, Sarker SD, Ghildyal R, Arya A (2019) Endoplasmic Reticulum Stress Activates Unfolded Protein Response Signaling and Mediates Inflammation, Obesity, and Cardiac Dysfunction: Therapeutic and Molecular Approach. Front Pharmacol 10: 977.

40. Tang CH, Ranatunga S, Kriss CL, Cubitt CL, Tao J, et al. (2014) Inhibition of ER stress-associated IRE-1/XBP-1 pathway reduces leukemic cell survival. J Clin Invest 124(6): 2585-2598.

41. Jin JK, Blackwood EA, Azizi K, Thuerauf DJ, Fahem AG, et al. (2017) ATF6 Decreases Myocardial Ischemia/Reperfusion Damage and Links ER Stress and Oxidative Stress Signaling Pathways in the Heart. Circ Res $120(5): 862-875$

42. Kim CY, Kim KH (2018) Selenate Prevents Adipogenesis through Induction of Selenoprotein S and Attenuation of Endoplasmic Reticulum Stress. Molecules 23(11).

43. Szegezdi E, Logue SE, Gorman AM, Samali A (2006) Mediators of endoplasmic reticulum stress-induced apoptosis. EMBO Rep 7(9): 880885.

44. Yin J, Gao Z, He Q, Zhou D, Guo Z, Ye J (2009) Role of hypoxia in obesityinduced disorders of glucose and lipid metabolism in adipose tissue. Am J Physiol Endocrinol Metab 296(2): E333-E342.

45. Zhu W, Niu X, Wang M, Li Z, Jiang HK, et al. (2019) Endoplasmic reticulum stress may be involved in insulin resistance and lipid metabolism disorders of the white adipose tissues induced by high-fat diet containing industrial trans-fatty acids. Diabetes Metab Syndr Obes 12: 1625-1638.

46. Sharma NK, Das SK, Mondal AK, Hackney OG, Chu WS, et al. (2008) Endoplasmic reticulum stress markers are associated with obesity in nondiabetic subjects. J Clin Endocrinol Metab 93(11): 4532-4541.

47. Haukeland JW, Dahl TB, Yndestad A, Gladhaug IP, Løberg EM, et al. (2012) Fetuin A in nonalcoholic fatty liver disease: in vivo and in vitro studies. Eur J Endocrinol 166(3): 503-510.

48. Abdullahi A, Jeschke MG (2016) White Adipose Tissue Browning: A Double-edged Sword. Trends Endocrinol Metab. 27(8): 542-552.

49. Shan B, Wang X, Wu Y, Xu C, Xia Z, et al. (2017) The metabolic ER stress sensor IRE1alpha suppresses alternative activation of macrophages and impairs energy expenditure in obesity. Nat Immunol 18(5): 519-529.

50. Liu Z, Gu H, Gan L, Xu Y, Feng F, et al. (2017) Reducing Smad3/ATF4 was essential for Sirt1 inhibiting ER stress-induced apoptosis in mice brown adipose tissue. Oncotarget 8(6): 9267-9279.

51. Graham RR, Kozyrev SV, Baechler EC, et al. (2006) A common haplotype of interferon regulatory factor 5 (IRF5) regulates splicing and expression and is associated with increased risk of systemic lupus erythematosus. Nat Genet 38(5): 550-555.

52. Bartelt A, Widenmaier SB, Schlein C, Plenge RM, Bauer JW, et al. (2018) Brown adipose tissue thermogenic adaptation requires Nrf1-mediated proteasomal activity. Nat Med 24(3): 292-303. 
53. Kulathu Y, Komander D (2012) Atypical ubiquitylation - the unexplored world of polyubiquitin beyond Lys48 and Lys63 linkages. Nat Rev Mol Cell Biol 13(8): 508-523.

54. Schroeter EH, Kisslinger JA, Kopan R (1998) Notch-1 signalling requires ligand-induced proteolytic release of intracellular domain. Nature 393(6683): 382-386.

55. Bi P, Shan T, Liu W, Yue F, Yang X, et al. (2014) Inhibition of Notch signaling promotes browning of white adipose tissue and ameliorates obesity. Nat Med 20(8): 911-918.

56. Dupuis S, Jouanguy E, Al-Hajjar S, Fieschi C, Al-Mohsen IZ, et al. (2003) Impaired response to interferon-alpha/beta and lethal viral disease in human STAT1 deficiency. Nat Genet 33(3): 388-391.

57. Lee K, Um SH, Rhee DK, Pyo S (2016) Interferon-alpha inhibits adipogenesis via regulation of JAK/STAT1 signaling. Biochim Biophys Acta 1860 (11 Pt A): 2416-2427.

58. Kissig M, Ishibashi J, Harms MJ, Lim HW, Stine RR, et al. (2017) PRDM16 represses the type I interferon response in adipocytes to promote mitochondrial and thermogenic programing. EMBO J 36(11): 15281542 .
59. Derecka M, Gornicka A, Koralov SB, Szczepanek K, Morgan M, et al. (2012) Tyk2 and Stat3 regulate brown adipose tissue differentiation and obesity. Cell Metab 16(6): 814-824.

60. Honda K, Taniguchi T (2006) IRFs: master regulators of signalling by Toll-like receptors and cytosolic pattern-recognition receptors. Nat Rev Immunol 6(9): 644-658.

61. Okla M, Wang W, Kang I, Pashaj A, Carr T, Chung S (2015) Activation of Toll-like receptor 4 (TLR4) attenuates adaptive thermogenesis via endoplasmic reticulum stress. J Biol Chem 290(44): 26476-26490.

62. Kumari M, Wang X, Lantier L, Lyubetskaya A, Eguchi J, et al. (2016) IRF3 promotes adipose inflammation and insulin resistance and represses browning. J Clin Invest 126(8): 2839-2854.

63. Nam S, Lim JS (2016) Essential role of interferon regulatory factor 4 (IRF4) in immune cell development. Arch Pharm Res 39(11): 15481555.

64. Kong X, Banks A, Liu T, et al. (2014) IRF4 is a key thermogenic transcriptional partner of PGC-1alpha. Cell 158(1): 69-83. 